\title{
Frequency and Etiology of Pancytopenia in Patients Admitted to a Tertiary Care Hospital in Karachi
}

\author{
Rabia Farooque ${ }^{1,2}$, Sundus Iftikhar ${ }^{3}$, Fivzia Herekar ${ }^{4}$, Muhammad Junaid Patel ${ }^{2}$ \\ 1. Internal Medicine, Liaquat University of Medical and Health Sciences, Jamshoro, PAK 2. Internal Medicine, The Indus \\ Hospital, Karachi, PAK 3. Statistics, Indus Hospital Research Center, The Indus Hospital, Karachi, PAK 4. Internal \\ Medicine and Infectious Diseases, The Indus Hospital, Karachi, PAK
}

Corresponding author: Rabia Farooque, rabia.farooque92@gmail.com

\section{Abstract}

\section{Introduction}

Pancytopenia is an important hematologic problem encountered frequently in clinical practice characterized by a reduction in all three peripheral blood cell lineages, i.e., anemia, leucopenia, and thrombocytopenia, caused by myriad disease processes. Our study aimed to determine the frequency and etiology of pancytopenia in patients admitted under internal medicine services in a tertiary care hospital.

\section{Method}

This cross-sectional study was conducted in the in-patient internal medicine department, The Indus Hospital (TIH), Karachi, included 258 patients. To be eligible, participants had to give informed consent, be 14 years or older, and of either sex. The study involved a 20-30-minute interaction with the patient, involving an interview and physical examination, and access to electronic health record data.

\section{Results}

Out of 258 patients studied, 24 (9.3\%) were diagnosed with pancytopenia, the male to female ratio was 1:1, no significant difference was observed in the proportion of ethnicity, religion, previous treatment, known infectious disease, and personal and occupational exposure among pancytopenic patients and other nonpancytopenic patients. Fever $(\mathrm{n}=14,58.3 \%)$ was most common presenting complaint followed by fatigue $(\mathrm{n}=13,54.2 \%)$ and weight loss $(\mathrm{n}=7,29.2 \%)$ while most common signs were pallor $(87.5 \% \mathrm{n}=21)$, hepatomegaly $(29.2 \%, n=7)$, and splenomegaly $(25 \%, n=6)$. The most common cause of pancytopenia was megaloblastic anemia $(n=10,41.7 \%)$, followed by hypersplenism $(n=4,16.6 \%)$, acute infectious diseases $(\mathrm{n}=3,12.5 \%)$, and autoimmune diseases $(\mathrm{n}=3,12.5 \%)$.

\section{Conclusion}

Review began 10/11/2020 Review ended 10/15/2020 Published 10/20/2020

\section{() Copyright 2020}

Farooque et al. This is an open access article distributed under the terms of the Creative Commons Attribution License CC-BY 4.0., which permits unrestricted use, distribution, and reproduction in any medium, provided the original author and source are credited.

Our study suggests that pancytopenia is a common finding among our patient population and a larger proportion has a treatable cause, thus carrying a favorable prognosis.

Categories: Internal Medicine, Hematology

Keywords: pancytopenia

\section{Introduction}

Pancytopenia is an important hematologic problem encountered frequently in clinical practice [1]. It's defined as a reduction in all three peripheral blood cell lineages, i.e., anemia, leucopenia, and thrombocytopenia [2]. It's not a disease entity itself but a presentation caused by diverse disease processes affecting bone marrow and/or peripheral cell lines [3-4]. Clinical presentation is related to the severity of cytopenias, leading to common presenting symptoms, including generalized weakness, shortness of breath, fever, weight loss, bleeding, etc. The management and prognosis of pancytopenia depend upon its severity and underlying etiology [5]. Its etiology ranges from benign conditions, such as nutritional deficiencies, infection, and drug effects, to malignant diseases such as lymphomas and leukemias [6]. Thus, identifying the correct etiology is of crucial importance in formulating therapeutic plans [7]. Its etiology is influenced by geography, socio-economic conditions, and endemic illnesses [8]. Nutritional megaloblastic anemia, caused by folate or vitamin B12 deficiency, is one of the leading causes of pancytopenia in developing countries, as it's readily correctable and so should be suspected in patients with unexplained pancytopenia, macrocytosis, hypersegmented neutrophils, and neurological signs and symptoms $[4,6]$. There's a scarcity of data regarding the prevalence of pancytopenia in Pakistan. Therefore, we aimed to determine the frequency of pancytopenia and to evaluate the clinical features and underlying etiology of pancytopenia in the adult population admitted under internal medicine services in a tertiary care hospital. 


\section{Materials And Methods}

This prospective cross-sectional study was conducted at The Indus Hospital, Karachi (TIH), a free-of-cost tertiary-care facility. A priori sample size of 258 was calculated using OpenEpi software (www.OpenEpi.com) with the following assumptions: prevalence of pancytopenia $21.2 \%$ [9], 5\% desired precision, and 95\% confidence interval. The institutional review board of The Indus Hospital approved all study protocols. Patients were recruited from the in-patient internal medicine department over a four-month period between July 2018 and October 2018. Patients admitted under internal medicine services at TIH, age 14 years and above, of either gender, and giving informed consent either themselves or their guardians if age was below 18 years were included in the study. Of 258 patients recruited from the internal medicine department, none was excluded, as no patient refused to participate in the study. Data on demographics, presenting symptoms, and medical history regarding known co-morbidities or chronic infections, exposure to potentially toxic agents or radiation, and medication use were recorded. Physical examination was performed to assess pallor, rash, oral lesions, jaundice, lymphadenopathy, hepatomegaly, or splenomegaly.

Pancytopenia was defined by the complete blood count report, as all peripheral blood lineages decreased below the normal reference range, based on criteria defined by De Gruchy [10] as follows:

Hemoglobin $(\mathrm{Hb})$ level - $<13.5 \mathrm{~g} / \mathrm{dL}$ for males and $<11.5 \mathrm{~g} / \mathrm{dL}$ for females

Total leucocyte count (TLC) $-<4 \times 10^{\wedge} 9 / \mathrm{L}$

Platelet (Plt) count - <150×10^9/L

Further workup to identify the etiology of pancytopenia was carried out as clinically indicated, which included reticulocyte count, serum lactate dehydrogenase (LDH), serum iron profile, vitamin B12 levels, red blood cell (RBC) folate, blood cultures, malarial parasite (MP), liver function test (LFT), chronic viral hepatitis serology, human immunodeficiency virus (HIV) screening serology, ultrasound and CT scan imaging, and bone marrow biopsy.

All the data were gathered on a predesigned questionnaire.

\section{Results}

A total of 258 patients were enrolled in the study with a median age of 48 (28.8-65) years. Out of these, the majority $(164 ; 63.6 \%)$ were females. One-hundred sixty-six (64.3\%) of the patients were not working,

followed by services and sales workers $(\mathrm{n}=88 ; 34.1 \%)$ and elementary occupation $(\mathrm{n}=34 ; 13.2 \%)$. Out of those who were not doing any kind of job, the majority were housewives ( $\mathrm{n}=94 ; 56.6 \%), 22(13.3 \%)$ were students, and 28 (16.9\%) were dependent/bed-bound (Table 1). 


\section{Cureus}

Variable

$n(\%)$

Gender

Female

Male

Age

Median (IQR)

Min-Max

Occupation

Not working

Services and sales workers

Elementary occupation

Craft and related trades workers

Plant and machinery operator

Professionals

Technicians and associate professionals

Skilled agricultural, forestry, and fishery workers

Clerical support workers

Forces

Reasons for not working

Housewife

Dependent/bed-bound

Student

Retired/pensioner

Unemployed
$164(63.6)$

94 (36.4)

$48(28.8-65)$

$14-100$

166 (64.3\%)

88 (34.1)

34 (13.2)

$14(5.4$

11 (4.3)

9 (3.5)

8 (3.1)

4 (1.6)

$1(0.4)$

$1(0.4)$

94 (56.6)

28 (16.9)

22 (13.3)

$16(9.6)$

6 (3.6)

\section{TABLE 1: Demographic data}

More than half of the patients had one or more co-morbidity, with hypertension $(n=95 ; 36.8 \%)$ and diabetes mellitus ( $n=67 ; 26 \%$ ) being the most commonly observed among the patients (Table 2).

\begin{tabular}{|c|c|}
\hline \multicolumn{2}{|l|}{ Known Co-morbid Diseases } \\
\hline None & $114(44.2)$ \\
\hline Hypertension (HTN) & $95(36.8)$ \\
\hline Diabetes mellitus (DM) & $67(26)$ \\
\hline Ischemic heart disease (IHD) & $35(13.6)$ \\
\hline Cerebrovascular accident (CVA) & $16(6.2)$ \\
\hline Chronic kidney disease (CDK) & $15(5.8)$ \\
\hline Connective tissue disease & $11(4.3)$ \\
\hline Chronic obstructive pulmonary disease (COPD) & $8(3.1)$ \\
\hline Chronic liver disease & $5(1.9)$ \\
\hline
\end{tabular}




\section{Cureus}

End-stage renal disease (ESRD)

$4(1.6)$

Known Psychiatric illness

$2(0.8)$

Malignancy

$2(0.8)$

Cirrhosis

$1(0.4)$

Inflammatory bowel disease (IBD)

$1(0.4)$

Hemoglobinopathies (Thalassemia)

$1(0.4)$

Known infectious diseases

None

$226(87.6)$

Tuberculosis

18 (7)

Chronic viral

$12(4.7)$

Human immunodeficiency virus (HIV)/acquired immunodeficiency syndrome (AIDS)

$2(0.8)$

Description of prescribed medication

None

$148(57.4)$

Angiotensin-converting enzyme inhibitors (ACE)/angiotensin receptor blocker (ARBs)

$41(15.9)$

Oral hypoglycemics

$36(14)$

Antiplatelet agents

$30(11.6)$

Beta-blockers

$23(8.9)$

Calcium channel blocker (CCB)

$20(7.8)$

Gastrointestinal (acid suppressants)

$14(5.4)$

Thiazide/loop diuretics

$12(4.7)$

Steroids

$11(4.3)$

Statin

$10(3.9)$

Vasodilators (nitrates/hydralazine)

$9(3.5)$

Disease-modifying anti-rheumatic drugs (DMARDs)

$7(2.7)$

Analgesics

Bronchodilators (inhaled/oral)

Anticoagulant agents

$3(1.2)$

Anti-thyroid

$3(1.2)$

Anti-epileptics

$3(1.2)$

Alpha-blockers

$3(1.2)$

Anti-depressants

$2(0.8)$

Sedatives/hypnotics

$2(0.8)$

Anxiolytics

$1(0.4)$

Anti-neoplastic drugs

$1(0.4)$

Anti-tuberculosis therapy

$1(0.4)$

Nonprescription medication

None

$199(77.1)$

Allopathic medication

$259.7)$ 


\section{Cureus}

\begin{tabular}{l|l|} 
Herbal medication & $25(9.7)$ \\
Homeopathic medication & $21(8.1)$ \\
Personal \& occupation exposure & $242(93.8)$ \\
None & $4(1.6)$ \\
Alcohol & $4(1.6)$ \\
Glue vapors & $3(1.2)$ \\
Organic solvents & $1(0.4)$ \\
Benzene & $1(0.4)$ \\
Radiation &
\end{tabular}

TABLE 2: Medical history

Furthermore, three-fifths of the patients had monocytopenia ( $\mathrm{n}=154 ; 59.7 \%), 47$ (18.2\%) had bicytopenia, and 24 (9.3\%) had pancytopenia. Out of 154 monocytopenic patients, 149 (96.8\%) had anemia while five (3.2\%) had thrombocytopenia and none of the patients had leucopenia (Table 3).

\begin{tabular}{|lr|}
\hline Cytopenia categories & \\
\hline Pancytopenia & $24(9.3)$ \\
\hline Bicytopenia & $47(18.2)$ \\
Monocytopenia & $154(59.7)$ \\
Normal & $33(12.8)$
\end{tabular}

TABLE 3: Cytopenia categories

Among pancytopenic patients, fever was the most common presenting complaint $(\mathrm{n}=14,58.3 \%)$ followed by fatigue $(n=13,54.2 \%)$ and weight loss $(n=7,29.2 \%)$. Pallor was seen in $87.5 \%(n=21)$ while hepatomegaly was found in $29.2 \%(n=7)$, splenomegaly in $25 \%(n=6)$, jaundice, rash and oral ulcers in $8.3 \%$ each $(n=2$ each), and lymphadenopathy in $4.2 \%(\mathrm{n}=1)$ (Table 4$)$. 


\section{Cureus}

\begin{tabular}{|l|l|}
\hline Clinical feature & Frequency (\%) \\
\hline Pallor & $21(87.5 \%)$ \\
Fever & $14(58.3 \%)$ \\
Fatigue & $13(54.2 \%)$ \\
Weight loss & $7(29.2 \%)$ \\
Hepatomegaly & $7(29.2 \%)$ \\
Splenomegaly & $6(25 \%)$ \\
Jaundice & $2(8.3 \%)$ \\
Rash & $2(8.3 \%)$ \\
Oral ulcers & $2(8.3 \%)$ \\
Lymphadenopathy & $1(4.2 \%)$ \\
\hline
\end{tabular}

\section{TABLE 4: Clinical features of pancytopenia}

The most common cause of pancytopenia was found to be megaloblastic anemia $(n=10,41.7 \%)$ followed by hypersplenism $(n=4,16.6 \%)$, acute infectious cause $(n=3,12.5 \%)$, and autoimmune diseases $(n=3,12.5 \%)$. Chronic Hodgkin lymphoma was diagnosed in one patient (4.1\%) and one patient was found to have chronic kidney disease (CKD). Two patients had pancytopenia with no obvious cause (Table 5).

\begin{tabular}{|l|l|}
\hline Causes of Pancytopenia & No. of cases (\%) \\
\hline Megaloblastic anemia & $10(41.7 \%)$ \\
Hypersplenism & $4(16.7 \%)$ \\
Acute infectious disease & $3(12.5 \%)$ \\
Autoimmune disease & $3(12.5 \%)$ \\
Chronic kidney disease & $1(4.2 \%)$ \\
Chronic Hodgkin lymphoma & $1(4.2 \%)$ \\
None & $2(8.3 \%)$ \\
\hline
\end{tabular}

\section{TABLE 5: Etiology of pancytopenia}

In the hypersplenism group, one patient had chronic liver disease (CLD) secondary to hepatitis C infection, two had non-B/C CLD, and one had isolated splenomegaly with no cause identified. The three patients had pancytopenia associated with infectious diseases; their etiology was fulminant hepatic failure along with hospital-acquired septicemia, enteric fever, and complicated malaria, respectively. While, in the autoimmune disease group, one patient had autoimmune hemolytic anemia, the second had small vessel vasculitis, and the third had systemic lupus erythematosus.

Additionally, the distribution of pancytopenia was equal in both the genders ( $\mathrm{p}=0.182$; Table 6 ), whereas, the proportion of B-12 deficiency was higher in pancytopenia patients as compared to the non-pancytopenia patients ( $40 \%$ vs $6.8 \%, \mathrm{p}=0.001$ ). No statistically significant association was seen between pancytopenia and ethnicity, religion, previous treatment, infectious disease, and personal and occupational exposure (Table 6).

\section{Pancytopenia}

No Yes Total

\section{P-value}




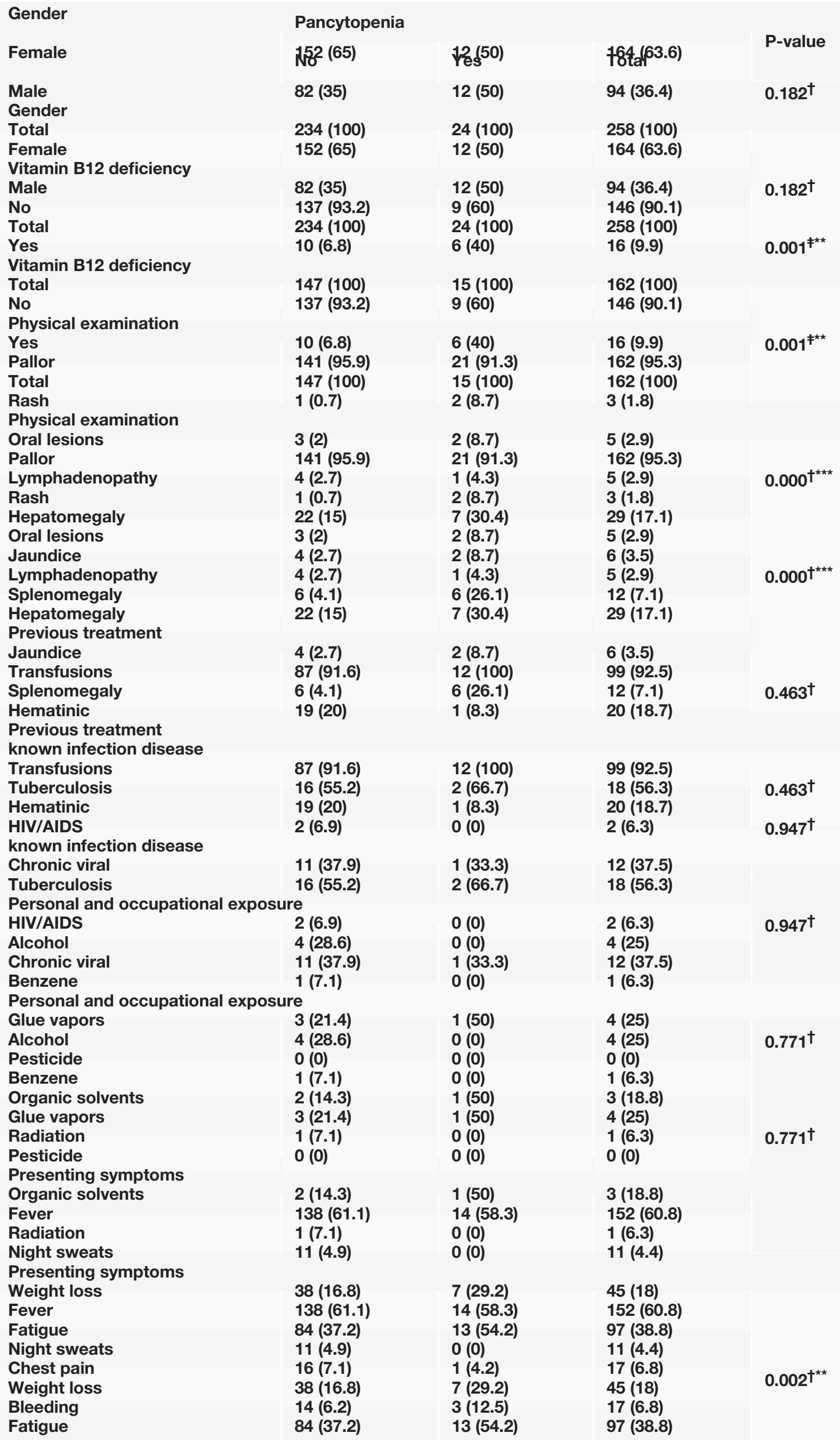




\begin{tabular}{|c|c|c|c|c|}
\hline \multirow{2}{*}{$\begin{array}{l}\text { Jaundice } \\
\text { Nausea/vomiting }\end{array}$} & 3dh'3)topenia & $4(16.7)$ & $7(2.8)$ & \multirow{2}{*}{ P-value } \\
\hline & $6(29.2)$ & उष20.8) & Tptal8.4) & \\
\hline Ghortness of breath & $12(5.3 \%)$ & $3(12.5 \%)$ & $15(6)$ & \\
\hline \multicolumn{4}{|c|}{ 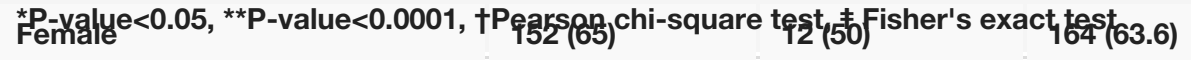 } & \multirow{3}{*}{$0.182^{\dagger}$} \\
\hline Male & $82(35)$ & $12(50)$ & $94(36.4)$ & \\
\hline \multicolumn{4}{|c|}{$\begin{array}{l}\text { TABLE 6: Association of pancytopenia with patients' characteristics } \\
\text { Total } 234(100) \\
248(100)\end{array}$} & \\
\hline
\end{tabular}

Vitamin B12 deficiency

No

Yes

Total

Physical examination

Pallor

Rash

Oral lesions

Lymphadenopathy

Hepatomegaly

Jaundice

Splenomegaly

Previous treatment

Transfusions

Hematinic

known infection disease

Tuberculosis

HIV/AIDS

Chronic viral four patients had hypersplenism because of liver cirrhosis, one had isolated splenomegaly with no cause

Out of 24 pancytopenic patients, 18 patients were discharged with follow-up advised in medical outpatient

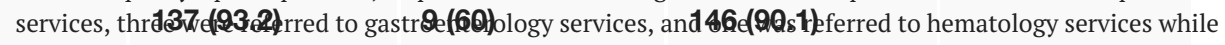
two patients expired during the hospital stay. $10(6.8)$
$6(40)$

\section{Discuspioitoo)}

15 (100)

$16(9.9)$

$0.001^{\text {**}^{*}}$

Pancytopenia is a hematologic entity frequently encountered in clinical practice. In this study, the incidence of pancytopenia was $9.3 \%$ in patients admitted under internal medicine services while in other studies, its frequency is quite yariable, and most of these studies are conducted on pediatric patients. A study conducted at Kuwait Teaching Hospital, Peshawar, showed its frequency to be $57.4 \%$ in the adult medicine department in 2015 [11] while 7 nother study conducted in the pathology department, Rawalpindi, Pakistan determined the frequency of pancytopenia in the combined adult and pediatric population to be $21.2 \%$ [9]. Umbreen et

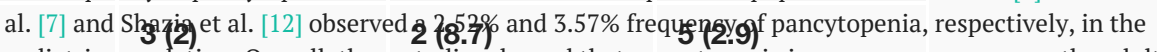
pediatric population. Overall, these studies showed that pancytopenia is more common among the adult population in P2kig tan.

1 (4.3)

5 (2.9)

$0.000^{\dagger^{* * *}}$

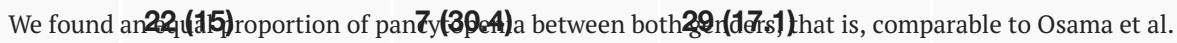
[13], whereas the Yaseen et al. study [3] showed that females constituted $62 \%$ of the pancytopenic

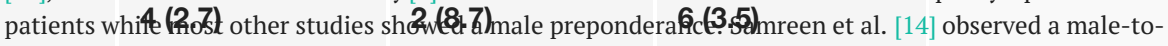
female ratio of 1.8:1 while Umbreen et al. [9 9 and Ikram et al [6] reported this to be 2.5:1 and 2:1, respectively. $\mathbf{6}(\mathbf{4 . 1 )} \quad \mathbf{6}(\mathbf{2 6 . 1 )} \quad \mathbf{1 2}(\mathbf{7 . 1 )}$

Furthermore, we observed that pancytopenic patients were younger, with a median age of 38.8 years, which

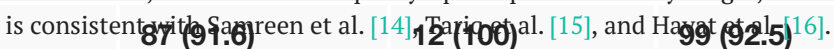

$0.463^{\dagger}$

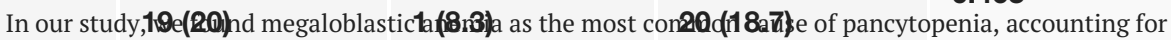
41.7\%. Osama et al. [13], Yaseen et al. [3], Aziz et al. [17], and studies from India had similar observations [5,18-21]. All patients in our study with megaloblastic anemia had a vitamin B 12 deficiency that is easily correctable, hence it should be suspected early on the basis of the megaloblastic picture on peripheral smear and mean colfu(55.2) volume above4d(69.7) 3]. 18 (56.3)

Hypersplenism accounted for $16.7 \%$ of cases of pancytopenia in this study while Hayat et a a. ${ }^{\dagger}$. [16], Osama et

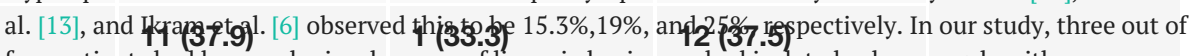

Personal and occupationailextifiostured only one had cirrhosis secondary to chronic hepatitis $\mathrm{C}$ infection. This is in contrast to the

Alcohol

4 (28.6)

$\mathbf{0}(0)$

4 (25)

Benzene

Glue vapors

Pesticide

Organic solvents

Radiation

Presenting symptoms

Fever

Night sweats

Weight loss

Fatigue

In our study, $12.5 \%$ of patients with pancytopenia had acute infectious etiology; while Osama et al. [13] and

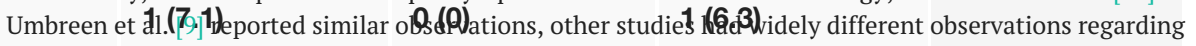
infectious etiology [1-2,5,14,23-25].

$$
\mathbf{3}(\mathbf{2 1 . 4 )} \mathbf{1} \text { (50) }
$$

4 (25)

Autoimmung digeases caused pancytop 0 ) ia in $12.5 \%$ of patiegtsoput of three, one patient had autoimmune hemolytic anemia, the second had small vessel vasculitis, and the third had systemic lupus erythematosus.

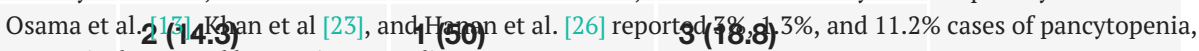
respectively, caused by autoimmune diseases.
1 (7.1)
0 (0)
1 (6.3)

Chronic Hodgkin lymphoma was diagnosed in one patient (4.1\%); other studies observed malignant conditions more frequently than this [2,9,13-14,16-17].

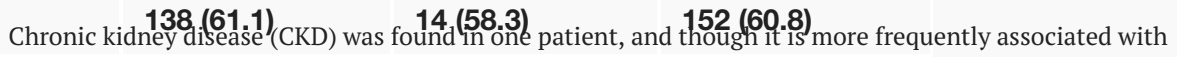

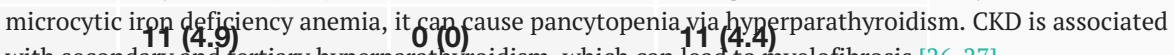
with secondary and tertiary hyperparathyroidism, which can lead to myelofibrosis [26-27].
38 (16.8)

Conclusions
7 (29.2)

$13(54.2)$
45 (18)

97 (38.8) 
In this study, we found that pancytopenia is a common finding among our adult population, and a larger proportion had a treatable cause, thus carrying a favorable prognosis. It also emphasizes the need for an accurate diagnosis that can facilitate timely treatment and impact morbidity and mortality.

\section{Additional Information \\ Disclosures}

Human subjects: Consent was obtained by all participants in this study. Interactive Research \& Development (IRD) - Institutional Review Board (IRB) issued approval IRD-IRB \# IRD_IRB_2018_05_011. IRB EXPEDITED STATUS: APPROVED The IRD-IRB has reviewed the above-referenced study and determined that, as currently described, it was eligible for expedited review and has been approved, as per the following category: Category 02: Collection of blood samples by finger stick, heel stick, ear stick, or venipuncture as follows: From other adults and children, considering the age, weight, and health of the subjects, the collection procedure, the amount of blood to be collected, and the frequency with which it will be collected. For these subjects, the amount drawn may not exceed the lesser of $50 \mathrm{ml}$ or $3 \mathrm{ml}$ per $\mathrm{kg}$ in an 8 week period and collection may not occur more frequently than 2 times per week. Animal subjects: All authors have confirmed that this study did not involve animal subjects or tissue. Conflicts of interest: In compliance with the ICMJE uniform disclosure form, all authors declare the following: Payment/services info: All authors have declared that no financial support was received from any organization for the submitted work. Financial relationships: All authors have declared that they have no financial relationships at present or within the previous three years with any organizations that might have an interest in the submitted work. Other relationships: All authors have declared that there are no other relationships or activities that could appear to have influenced the submitted work.

\section{Acknowledgements}

The authors wish to thank the participants, staff of Indus Hospital Research Center (IHRC), and Dr. Naila Baig-Ansari, chair-IHRC, for their support of this study.

\section{References}

1. Gayathri B, Rao KS: Pancytopenia: a clinico hematological study . J Lab Physicians. 2011, 3:15-20. 10.4103/0974-2727.78555

2. Jain A, Naniwadekar M: An etiological reappraisal of pancytopenia-largest series reported to date from a single tertiary care teaching hospital. BMC Blood Disord. 2013, 13:10. 10.1186/2052-1839-13-10

3. Yaseen Khan SA, Khan I, Taqveem A, Awan B: Frequency of aplastic anemia and megaloplastic anemia as causes of pancytopenia in adults. KJMS. 2018, 11:72.

4. Gnanaraj J, Parnes A, Francis CW, Go RS, Takemoto CM, Hashmi SK: Approach to pancytopenia: diagnostic algorithm for clinical hematologists. Blood Rev. 2018, 32:361-367. 10.1016/j.blre.2018.03.001

5. Kumar V, Khare M, Kishore M, Sharma M, Marwah S, Nigam AS, Singh P: Diagnostic approach of new-onset pancytopenia: study from a tertiary care center. Annals Pathol Lab Med. 2018, 5:[Epub]. 10.21276/APALM.1875

6. Ujjan I, Shaikh I, Khokhar NA, Memon R, Farooq M: Frequency of causes of pancytopenia in patients admitted at Isra University Hospital Hyderabad. Pak J Med Health Sci. 2010, 4:416-418.

7. Ahmad A, Idrees M, Afridi IG, Rehman G: To determine etiology and frequency of pancytopenia in pediatric population and compare it with other studies. Khyber J Med Sci. 2016, 9:186-189.

8. Rehmani THR, Arif M, Heraid S, Arif S, Ahmad R, Saeed M: Spectrum of pancytopenia. A tertiary care experience. Prof Med J. 2016, 23:620-626. 10.17957/TPMJ/16.3398

9. Arshad U, Latif RK, Ahmad SQ, Imran MM, Khan F, Jamal S: Clinical and aetiological spectrum of pancytopenia in a tertiary care hospital. Pak Armed Forces Med J. 2016, 66:323-327.

10. Firkin F, Chesterman C, Penington D, Rush B: Pancytopenia and aplastic anaemia. de Gruchy's Clinical Haematology in Medical Practice, 5th Edition. Blackwell Scientific Publications, London, United Kingdom; 1989. 119-136.

11. Mehboob S, Shah F, Muhammad S, Shah IA, Zarin A: Etiological spectrum of cytopenias in adult Pakistani population: a single centre experience. Khyber Med Univ J. 2017, 9:188-191.

12. Memon S, Shaikh S, Nizamani M: Etiological spectrum of pancytopenia based on bone marrow examination in children. J Coll Physicians Surg Pak. 2008, 18:163-167.

13. Ishtiaq O, Baqai HZ, Anwer F, Hussain N: Patterns of pancytopenia patients in a general medical ward and a proposed diagnostic approach. J Ayub Med Coll Abbottabad. 2004, 16:8-13.

14. Samreen Z, Durrani AB, Taj MK: Frequency of common etiologies of pancytopenia seen on bone marrow aspiration. J Saidu Med Col. 2020, 9:178-181.

15. Tariq M, Basri R, Khan NU, Amin S: Aetiology of pancytopenia. Professional Med J. 2010, 17:252-256.

16. Hayat AS, Khan AH, Baloch GH, Shaikh N: Pancytopenia. Professional Med J. 2014, 21:60-65.

17. Aziz T, Ali L, Ansari T, Liaquat HB, Shah S, Ara J: Pancytopenia: megaloblastic anemia is still the commonest cause. Pak J Med Sci. 2010, 26:132-136.

18. Goli N, Koguru S, Wadia RS, et al.: Etiological profile of pancytopenia in a tertiary care hospital . Int J Adv Med. 2016, 3:533. http://dx.doi.org/10.18203/2349-3933.ijam20162188

19. Gore CR, Bardapurkar P, Paranjape S, Patel S, Karia K: Clinico-hematological evaluation of pancytopenic adults in a tertiary care. Indian J Pathol Oncol. 2018, 2:391-397. 10.18231/2394-6792.2018.0076

20. Govindaraj T, Rathna S, Venkatraman J: Bone marrow study in pancytopenia . Int J Cur Res Rev. 2015, 7:5052 .

21. Makheja KD, Maheshwari BK, Arain S, Kumar S, Kumari S: The common causes leading to pancytopenia in 
patients presenting to tertiary care hospital. Pak J Med Sci. 2013, 29:1108.

http://dx.doi.org/10.12669/pjms.295.3458

22. Asif AF: Appraisal of national response to chronic hepatitis in Pakistan . J Islamabad Med Dent College. 2019, 8:3-7.

23. Khan MI, Fatima SH, Ahmad N: Etiological spectrum of pancytopenia using bone marrow aspiration and biopsy. Pak J Pathol. 2017, 28:164-168.

24. Pathak R, Jha A, Sayami G: Evaluation of bone marrow in patients with pancytopenia . J Pathol Nepal. 2012, 2:265-271.

25. Tareen SM, Bajwa MA, Tariq MM, Babar S, Tareen AM: Pancytopenia in two national ethnic groups of Baluchistan. J Ayub Med Coll Abbottabad. 2011, 23:82-86.

26. El-Hagrasy HA, Hassanein N, Ahmed AME: Clinico-pathological study of pancytopenia in adult cases at a tertiary hospital in Saudi Arabia. Al-Azhar Assiut Med J. 2015, 13:1.

27. Nomura S, Ogawa Y, Osawa G, Katagiri M, Harada T, Nagahana H: Myelofibrosis secondary to renal osteodystrophy. Nephron. 1996, 72:683-687. 10.1159/000188961 